\title{
EGFR-dependent mechanisms of resistance to osimertinib determined by ctDNA NGS analysis identify patients with better outcome
}

\author{
Julie A. Vendrell ${ }^{1 \wedge}$, Xavier Quantin ${ }^{2}$, Audrey Aussel $^{1}$, Isabelle Solassol ${ }^{3}$, Isabelle Serre ${ }^{1}$, \\ Jérôme Solassol ${ }^{1,2}$
}

${ }^{1}$ CHU Montpellier, Department of Pathology and Onco-biology, Univ Montpellier, Montpellier, France; ${ }^{2}$ IRCM, INSERM, Univ Montpellier, ICM, Montpellier, France; ${ }^{3}$ IURT, ICM, Montpellier, France

Contributions: (I) Conception and design: JA Vendrell, J Solassol; (II) Administrative support: None; (III) Provision of study materials or patients: X Quantin, A Aussel, I Serre, J Solassol; (IV) Collection and assembly of data: JA Vendrell, I Solassol, J Solassol; (V) Data analysis and interpretation: JA Vendrell, I Solassol, J Solassol; (VI) Manuscript writing: All authors; (VII) Final approval of manuscript: All authors.

Correspondence to: Prof. Jérôme Solassol. Laboratoire de Biologie des Tumeurs Solides, Hôpital Arnaud de Villeneuve, CHU de Montpellier, 371, avenue du Doyen Gaston Giraud, 34295 Montpellier cedex 5, France. Email: j-solassol@chu-montpellier.fr.

Background: Osimertinib is an epidermal growth factor receptor (EGFR) tyrosine kinase inhibitor (TKI) that is highly selective for $E G F R^{\mathrm{T} 790 \mathrm{M}}$ subclones in patients with $E G F R^{\text {sensitizing }}$ non-small cell lung cancer (NSCLC). Unfortunately, all patients develop resistance through EGFR-dependent or EGFR-independent pathways. Recently, circulating tumoral DNA (ctDNA) analysis has highlighted the usefulness of plasma genotyping for exploring patient survival outcomes after disease progression under osimertinib.

Methods: Plasma samples from patients treated with osimertinib as a second-line therapy were collected and the presence of molecular alterations of acquired resistance was evaluated after relapse under osimertinib using ctDNA molecular profiling by next-generation sequencing (NGS) assays. The clinical implications of these genomic alterations for the efficiency of the third-generation TKI were further assessed.

Results: Our ctDNA molecular profiling of plasma samples highlighted large number of actionable genomic alterations. According to ctDNA NGS results, patients were classified as having developed an EGFR-dependent or EGFR-independent mechanism of resistance. Thus, patients who developed an EGFRdependent mechanism of resistance responded longer to osimertinib (13.8 vs. 4.6 months; $\mathrm{P}<10^{-4}$ ) and have a better post-osimertinib clinical outcome than EGFR-independent resistant patients. Moreover, the development of an EGFR-dependent mechanism of osimertinib resistance was identified as the best fit to determine patients' clinical outcome compared with $E G F R^{\mathrm{T} 790 \mathrm{M}}$ status alone $(\mathrm{P}=0.003)$.

Conclusions: Our study highlights the potential of ctDNA NGS to rapidly select the appropriate drug after osimertinib failure and to determine clinical outcomes of patients. We suggest that ctDNA NGS should be more intensively used in clinical practice to follow patients under third-generation TKIs.

Keywords: EGFR-TKI resistance; osimertinib; circulating tumor DNA; lung cancer; clinical outcome

Submitted Aug 26, 2021. Accepted for publication Oct 25, 2021.

doi: $10.21037 /$ tlcr-21-679

View this article at: https://dx.doi.org/10.21037/tlcr-21-679

^ ORCID: 0000-0002-2071-6634. 


\section{Introduction}

In patients with non-small cell lung cancer (NSCLC), sensitizing mutations in epidermal growth factor receptor $(E G F R)$ distinguish subgroups of patients with tumors that have higher sensitivity to tyrosine kinase inhibitors (TKIs) than tumors harboring wild-type EGFR. Osimertinib is a third-generation irreversible EGFR TKI that selectively targets $E G F R^{\text {activating }}$ mutations as well as the $E G F R^{\mathrm{T} 790 \mathrm{M}}$ mutation that is known as a common resistance mechanism of first- and second-generation TKIs. Due to positive clinical results of a phase 3 trial (FLAURA) comparing third-generation TKIs to standard TKIs in terms of progression-free survival (PFS), osimertinib was approved in 2018 as a first-line therapy for NSCLC carrying an $E G F R^{\text {activating }}$ mutation, but also in any line of therapy in patients with the $E G F R^{\mathrm{T} 790 \mathrm{M}}$ mutation $(1,2)$.

Unfortunately, acquired resistance after first- or secondline therapy with osimertinib emerges after a median response duration of 20.9 months and 9.9 to 12.3 months, respectively (3-5). To overcome resistance, several molecular studies have been performed. Most have used tissue samples obtained by new tumoral biopsy under osimertinib progression (6-9). However, tissue samples are often insufficient for molecular testing and do not systematically represent the whole mutational status of the patient. Cell-free DNA (cfDNA) and more precisely circulating tumor DNA (ctDNA) represents an attractive alternative to tissue sampling, notably during disease progression. Several studies have been reported but most employed approaches that limited the number of molecular alterations that could be detected, as they used single-target assays such as droplet digital PCR (ddPCR) or the Cobas ${ }^{\circledR}$ mutation test (10-16). Taking advantage of the breadth of coverage offered by next-generation sequencing (NGS)based approaches to detect genomic alterations in a single assay, we analyzed plasma ctDNA samples from a prospective observational cohort of 22 unselected patients treated with osimertinib as a second-line TKI. Samples were collected at patient relapse to assess diversity of acquired alterations under osimertinib and to evaluate third-line treatment efficiency.

We present the following article in accordance with the MDAR reporting checklist (available at https://dx.doi. org/10.21037/tlcr-21-679).

\section{Methods}

\section{Patients and sample collection}

For this observational study, 22 patients followed at the
University Hospital of Montpellier (France) for a stage IV NSCLC tumor harboring an EGFR mutation were prospectively enrolled from January 2017 to October 2020. All patients were initially treated with a first- or secondgeneration TKI and received osimertinib at relapse. The study was approved by the Institutional Review Board of the University Hospital of Montpellier (IRBMTP_2020_09_202000581). The study was conducted in accordance with the Declaration of Helsinki (as revised in 2013). An approved informed consent statement was acquired for all patients. Tissue and blood samples were collected at different points over the time-course treatment. For each patient, tissue samples were collected at cancer diagnosis. After standard pathological examination, tissue punches using a $1-\mathrm{mm}$ needle or macrodissected $10-\mu \mathrm{m}-$ thick section were performed from tumor paraffin blocks to increase the percentage of tumor cells in the sample, and analyzed for mutation detection by conventional approaches (high-resolution melting and/or Sanger sequencing). For each patient, blood samples were collected in cell-free DNA blood collection tubes (Streck, La Vista, NE, USA) at relapse under osimertinib. Medical records were reviewed to extract clinicopathological data, including sex, age, smoking status, diagnoses, therapeutic agents, and survival (Table 1). Tumor progression was defined according to RECIST 1.1 criteria (17).

\section{Cell-free DNA isolation}

cfDNA was isolated from blood samples using the QIAamp Circulating Nucleic Acid Kit (Qiagen, Hilden, Germany) and its integrity was checked using the D5000 ScreenTapes and a 4200 TapeStation instrument (Agilent Technologies, Santa Clara, CA, USA). DNA was quantified using the Qubit dsDNA BR Assay Kit and a Qubit Fluorometer (Thermo Scientific, Wilmington, DE, USA).

\section{ctDNA NGS analysis}

Libraries were prepared using the LiquidPlex ${ }^{\mathrm{TM}} 28$-gene Kit (Table S1, ArcherDX, Boulder, CO, USA) according to the manufacturer's instructions. The optimal input amount of double-stranded ctDNA was $50 \mathrm{ng}$. For samples yielding $<50 \mathrm{ng}$ of cfDNA, the entire amount was used for library preparation. Briefly, unamplified cfDNA molecules were ligated to adapters, unique molecule barcodes and a synthetic universal priming sequence, to enable target enrichment using gene-specific primers. After purification 
Table 1 Patient and specimen characteristics $(n=22)$

\begin{tabular}{|c|c|c|}
\hline Characteristics & $\mathrm{n}$ & $\%$ \\
\hline \multicolumn{3}{|l|}{ Sex } \\
\hline Male & 10 & 45.4 \\
\hline Female & 12 & 54.5 \\
\hline \multicolumn{3}{|l|}{ Age at diagnosis } \\
\hline$<60$ & 11 & 50.0 \\
\hline$\geq 60$ & 11 & 50.0 \\
\hline \multicolumn{3}{|l|}{ Smoking status } \\
\hline Have smoked & 5 & 22.7 \\
\hline Smoker & 0 & 0 \\
\hline Non-smoker & 12 & 54.5 \\
\hline Unknown & 5 & 22.7 \\
\hline \multicolumn{3}{|c|}{$E G F R^{\text {activating }}$ mutation status at diagnosis } \\
\hline$E G F R^{\text {del19 }}$ & 11 & 50.0 \\
\hline$E G F R^{\mathrm{L} 858 \mathrm{R}}$ & 9 & 40.9 \\
\hline$E G F R^{\mathrm{L} 861 \mathrm{Q}}$ & 3 & 13.6 \\
\hline \multicolumn{3}{|l|}{$1^{\text {st }}$ line TKI treatment } \\
\hline $1^{\text {st }}$ generation TKI & 14 & 63.6 \\
\hline $2^{\text {nd }}$ generation TKI & 8 & 36.4 \\
\hline \multicolumn{3}{|c|}{ Presence of metastases at osimertinib treatment initiation } \\
\hline Yes & 16 & 72.7 \\
\hline Bone & 13 & - \\
\hline Hepathic & 5 & - \\
\hline CNS & 6 & - \\
\hline Other sites & 2 & \\
\hline No & 4 & 18.2 \\
\hline Unknown & 2 & 9.1 \\
\hline \multicolumn{3}{|c|}{ Presence of metastases after relapse under osimertinib } \\
\hline Yes & 17 & 77.3 \\
\hline Bone & 14 & - \\
\hline Hepathic & 5 & - \\
\hline CNS & 5 & - \\
\hline Other sites & 3 & - \\
\hline No & 3 & 13.6 \\
\hline Unknown & 2 & 9.1 \\
\hline
\end{tabular}

EGFR, epidermal growth factor receptor; TKI, tyrosine kinase inhibitor; CNS, central nervous system. using Agencourt ${ }^{\circledR}$ AMPure ${ }^{\circledR}$ XP beads, a second PCR reaction was performed. After another purification step, libraries were quantified using the KAPA Library Quantification Kit (Roche, Meylan, France) and a LC480 instrument (Roche), normalized, pooled to equimolar concentration, and pair-end sequenced on an NextSeq (Illumina) instrument. Results were analyzed using the Archer Analysis v6.0.3.2 software. EGFR ${ }^{\text {activating }}$ patients were classified in two groups: (I) as having developed an EGFR-dependent mechanism if the $E G F R^{\mathrm{T} 790 \mathrm{M}}$ and/or any additional EGFR mutation were detected in the plasma sample; (II) as having developed an EGFR-independent mechanism if the $E G F R^{\mathrm{T} 790 \mathrm{M}}$ and not any other $E G F R$ mutation were reported.

\section{Statistical analysis}

PFS refers to first-line TKI treatment and was the time between the initiation of first-line TKI treatment and first patient relapse. Time to treatment discontinuation (TTD) refers to second-line TKI treatment and was defined as interval between the initiation to the end of osimertinib treatment (13). TTD2 refers to third-line treatment and was defined as interval between the initiation of chemotherapy and death or last visit. Overall survival (OS) was the time between cancer diagnosis and death or last visit. TTD, TTD2 and OS were estimated using the Kaplan-Meier method, and the significance of differences between survival rates was ascertained with the log-rank test using SPSS ${ }^{\circledR}$ Software (SPSS Inc., Chicago, IL, USA). A likelihood ratio test was applied to select the best fit between models significant in univariate analysis. $\mathrm{P}<0.05$ was considered significant.

\section{Results}

\section{Patients and clinical characteristics}

Twenty-two patients with advanced lung adenocarcinoma harboring an $E G F R^{\text {activating }}$ mutation and treated with TKI as first-line treatment were included in the study. The median follow-up was 44.9 months. The baseline characteristics of these patients at diagnosis are summarized in Table 1. The median age at diagnosis was 62 years old (range $33-76$ years), with slightly more women $(\mathrm{n}=12 ; 54.5 \%)$ than men, and non-smokers $(\mathrm{n}=12 ; 54.5 \%)$ than smokers. $50.0 \%(n=11)$ of the cases harbored at diagnosis an exon 19 
A

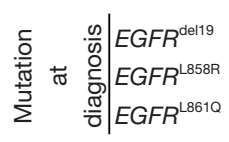

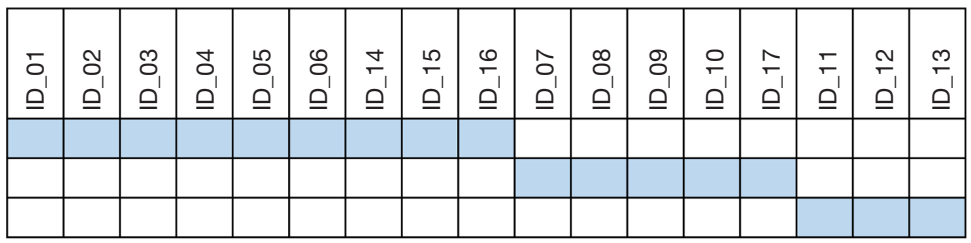

B

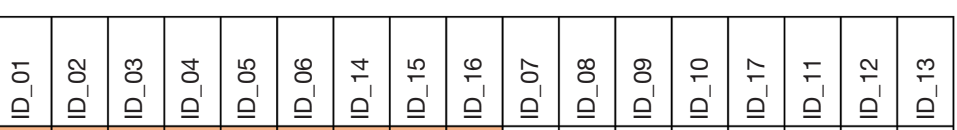

$\sum_{\infty} \mid \begin{aligned} & E G F R^{\text {del19 }} \\ & E G F R^{L 858 R} \\ & E G F R^{L 861 Q} \\ & E G F R^{T 790 M} \\ & E G F R^{\mathrm{C} 797 \mathrm{~S}} \\ & E G F R^{\mathrm{G} 796 \mathrm{~S}} \\ & E G F R^{L 718 Q} \\ & E G F R^{\mathrm{E} 788 \mathrm{D}} \\ & E G F R^{\mathrm{A} 559 \mathrm{~S}} \\ & E R B B 2^{\mathrm{G} 746 \mathrm{delinsvC}} \\ & B R A F^{\mathrm{V} 600 \mathrm{E}} \\ & K R A S^{\mathrm{G} 12 C} \\ & P I K 3 C A^{\mathrm{E} 545 \mathrm{~K}} \\ & T P 53 \text { non functional }\end{aligned}$
\begin{tabular}{lll|l|l|l|l|l|l|l|l|l}
3.8 & 6.0 & 5.8 & 21.8 & 1.2 & 4.8 & 29.6 & 8.1 & 0.9 \\
\hline
\end{tabular} \begin{tabular}{l|l|l|l|l|l}
12.8 & 75.0 & 4.0 & 0.7 & 0.4
\end{tabular}

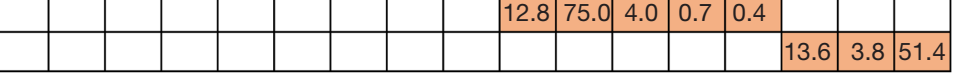

\begin{tabular}{|l|l|l|l|l|l|l|l|l|l|l|l|l|l|l|l|l|}
\hline 1.9 & 0.5 & 1.5 & & 0.4 & & & & & & 59.7 & & & & & 0.8 & \\
\hline & & & 5.4 & & & & & & 10.6 & & & & & & 0.9 & \\
\hline & & & & & & & & & & 31.3 & & & & & & \\
\hline & & & & & & & & & 0.8 & & 1.2 & & & & & \\
\hline & & 4.9 & & & & & & & & & & & & & & \\
\hline & & & & & & & & & & & & 0.8 & & & & \\
\hline & & & & & & & & & & & & & & 13.9 & & \\
\hline & 0.8 & & & & & & & & & & & & & & & \\
\hline & & & 3.3 & & & & & & & & & & & 0.7 & & \\
\hline & & & & & & 6.1 & & & & & & & & & & \\
\hline & 0.4 & & 10.7 & 2.0 & & 0.3 & 6.3 & 0.4 & 0.9 & 1.3 & 1.3 & & 0.3 & 6.8 & & \\
\hline
\end{tabular}

$$
\gtreqless \mid \begin{aligned}
& M E T \\
& B R A F \\
& \text { KRAS }
\end{aligned}
$$

\begin{tabular}{|l|l|l|l|l|l|l|l|l|l|l|l|l|l|l|l|l|}
\hline & & & & & 4.5 & & & & & & & & & & & 3.0 \\
\hline & & & & & & & & & & & & & & & & 3.5 \\
\hline & 4.0 & & & & & & & & & & & & & & & \\
\hline
\end{tabular}

Histological switch

Figure 1 Molecular alterations detected on tissue biopsy at diagnosis (A) and by NGS on ctDNA after relapse under osimertinib treatment (B). The numbers in the boxes correspond to the VAF of the mutation or the gene copy number for amplifications. SNV, Single nucleotide variant; $\mathrm{CNV}$, copy number variation.

deletion $\left(E G F R^{\text {del19 }}\right), 40.9 \%(\mathrm{n}=9)$ a p.L858R $\left(E G F R^{\mathrm{L} 858 \mathrm{R}}\right)$ and $13.6 \%(\mathrm{n}=3)$ a p.L861Q $\left(E G F R^{\mathrm{L} 861 \mathrm{Q}}\right)$.

All patients firstly received first- $(63.6 \%)$ or second(36.4\%) generation EGFR TKI treatment. The median duration of first-line treatment was 15.7 months. No significant difference was noticed in term of PFS and OS depending on the use of first- or second-generation TKI treatment $(\mathrm{P}=0.25$ and $\mathrm{P}=0.23$, respectively, Figure $\mathrm{S} 1)$.

After clinical evidence of relapse, confirmed by detection of an $E G F R^{\mathrm{T} 790 \mathrm{M}}$ mutation from tumor tissue and/or liquid biopsies, all patients received osimertinib as second-line treatment. At osimertinib introduction, 16 patients $(72.7 \%)$ had tumor involvement on metastatic sites. The median follow-up after osimertinib introduction was 27.2 months.

\section{Liquid biopsy molecular profiling}

For all patients, a liquid biopsy was systematically performed at progression under osimertinib treatment. Acquisition of co-occurring pathological alterations to $E G F R^{\text {activating }}$ was determined for all patients from ctDNA samples analyzed by NGS using a large panel of 28 genes. For five samples, no mutations were detected. As we could not exclude the absence of ctDNA in these specimens, they were removed from the study.

For the remaining 17 samples, a summary of the molecular alterations detected by NGS in the tissue before osimertinib treatment and the ctDNA samples at relapse are presented in Figure 1. The EGFR ${ }^{\text {activating }}$ mutation initially reported at tumor diagnosis was detected in all 
Table 2 Univariate analysis of the prognostic value of alterations detected by ctDNA NGS and clinical parameters with regard to TTD

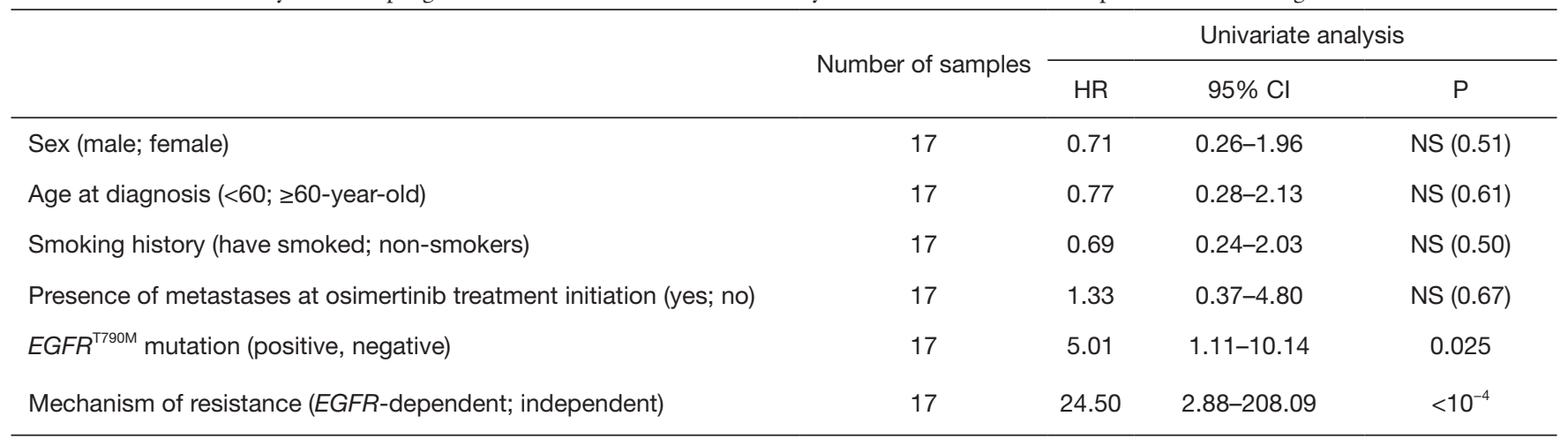

Significance (log-rank test) was considered at $\mathrm{P}<0.05$. ctDNA, circulating tumor DNA; NGS, next-generation sequencing; TTD, time to treatment discontinuation; NS, not significant; HR, hazard ratio; $95 \% \mathrm{Cl}, 95 \%$ confidence interval; EGFR, epidermal growth factor receptor.

samples. Nine patients also harbored one or two additional mutations in EGFR: $E G F R^{\mathrm{T} 790 \mathrm{M}}$ was detected in six samples (35.3\% of cases), $E G F R^{\mathrm{C} 797 \mathrm{~S}}$ in three samples $(17.6 \%$ of cases), and $E G F R^{\mathrm{L} 718 \mathrm{Q}}, E G F R^{\mathrm{E} 758 \mathrm{D}}, E G F R^{\mathrm{A} 859 \mathrm{~S}}$ and $E G F R^{\mathrm{G} 796 \mathrm{~S}}$ in one sample each (5.9\% of cases). Of note, $E G F R^{\mathrm{C} 7975}$ and $E G F R^{\mathrm{G} 796 \mathrm{~S}}$ detected in samples ID_08 and ID_12, respectively, were detected in cis of $E G F R^{\mathrm{T} 790 \mathrm{M}}$, meaning that they are harbored by the same allele.

Five patients (29.4\%) exhibited an actionable alteration in a gene other than $E G F R$. A $B R A F^{\mathrm{V} 600 \mathrm{E}}$ mutation (one patient), a PI3KCA $A^{\mathrm{E} 545 \mathrm{~K}}$ (one patient) and a $M E T$ amplification (two patients, $11.7 \%)$ were reported. Two patients harbored a mutation of resistance, $K R A S^{\mathrm{G} 12 \mathrm{C}}$. Additional gene amplifications affecting $B R A F$ and $K R A S$ genes were also found for one patient (5.9\%). As expected, the most altered gene in our cohort was TP53, for which mutations that induce a non-functional protein were reported in 11 patients (64.7\%). Finally, for four patients, relapse under osimertinib was explained by histologic transformation of advanced NSCLC to small cell lung cancer ( $\mathrm{n}=3$; ID_14; ID_16 and ID_17) or squamous cell carcinoma ( $\mathrm{n}=1$; ID_15), detected by immunohistochemistry on tissue biopsy analysis. For these 4 patients, an additional test consisting in $R B 1$ mutation detection was performed, resulting in the identification of a complete inactivation of $R B 1$ in 3 out of 4 patients.

\section{Patients who developed an EGFR-dependent mechanism of resistance responded for longer to osimertinib}

As detection of the $E G F R^{\mathrm{T} 790 \mathrm{M}}$ at relapse under osimertinib is a marker of good prognosis $(13,18)$, we first assessed the clinical outcome of patients harboring this alteration detected by NGS on ctDNA in term of TTD. In our cohort, patients with maintained $E G F R^{\mathrm{T} 790 \mathrm{M}}$ had a longer median TTD (17.6 months) than patients who lost it (9.0 months) $(P=0.025, \log$-rank test; Table 2 and Figure $2 A$ ). In line with recently published studies (19-21), at relapse, patients harboring an EGFR mutation in addition to $E G F R^{\text {activating }}$ already present at the initiation of the osimertinib treatment were classified as having an EGFR-dependent mechanism of resistance to osimertinib (58.8\%). By contrast, patients presenting a histological transformation or a mutation in an alternative pathway were classified as having an EGFR-independent mechanism $(41.2 \%)$. We found that patients who developed an EGFR-dependent mechanism of resistance responded for significantly longer to osimertinib (median $=13.8$ months) than patients who developed an EGFR-independent mechanism (median $=4.6$ months $)\left(\mathrm{P}<10^{-4} ; \log -\mathrm{rank}\right.$ test; Figure 2B,2C and Table 2). No prognostic value was observed by univariate analysis for the clinical parameters evaluated (Table 1), and no association was found between clinical characteristics and the mechanism of resistance developed by patients (Table S2). Moreover, the EGFR mechanism of resistance identified after osimertinib relapse was not associated to the first-line TKI treatment administrated to the patients $(\mathrm{P}=0.59$, Table S2). Of most interest, we found that the model based on an EGFRdependent mechanism of resistance was a better fit (likelihood $=41.16$ ) than the model with $E G F R^{\mathrm{T} 790 \mathrm{M}}$ only (likelihood $=50.19, \mathrm{P}=0.003$ ), showing that taking into account all of the mutations detected by NGS analysis to evaluate the mechanism of resistance involved provides the best prognostic value. 
A

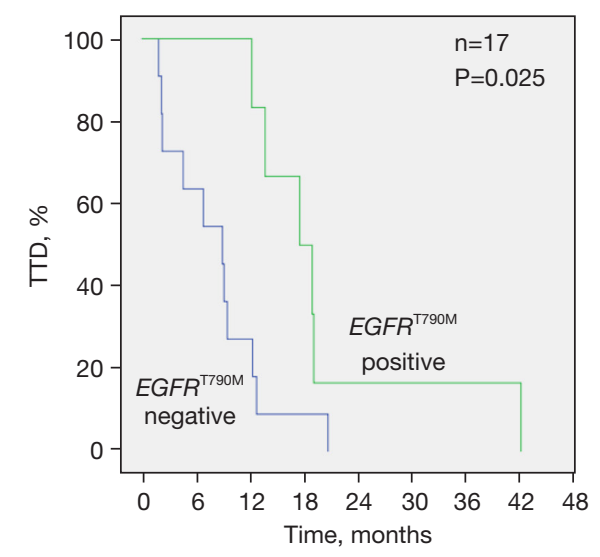

B

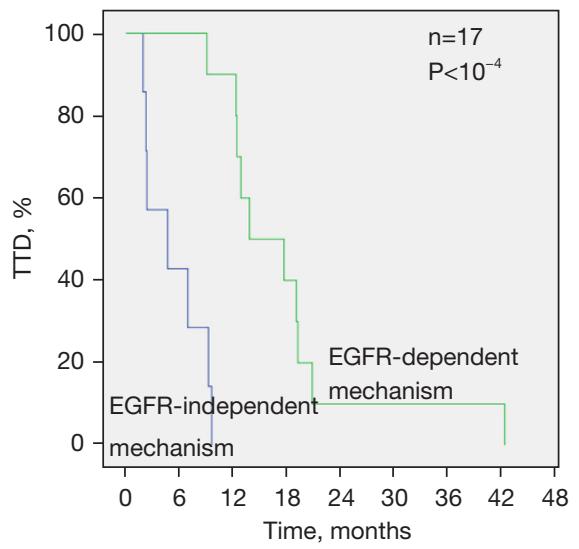

C

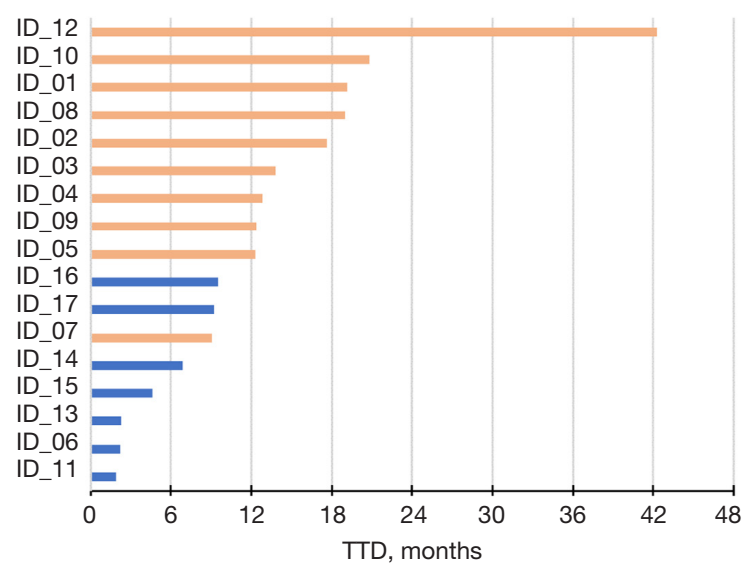

Figure 2 Clinical outcome of patients based on the alterations detected by ctDNA analysis. Kaplan-Meier analyses of patients with the $E G F R^{T 790 \mathrm{M}}$ mutation detectable at relapse by ctDNA NGS analysis (A), and of patients that have developed an EGFR-dependent mechanism of resistance (B). (C) Chart representing the individual response times to osimertinib treatment (in months). The orange bars represented patients who developed an EGFR-dependent mechanism of resistance and the blue bars, patients who developed an EGFR-independent mechanism. TTD, time to treatment discontinuation; ctDNA, circulating tumor DNA; NGS, next-generation sequencing; TTD, time to treatment discontinuation; EGFR, epidermal growth factor receptor.

\section{Post-osimertinib clinical outcome}

After relapse, all patients were treated with a third-line chemotherapy. We therefore investigated the clinical response of patients to this therapy according to the alterations detected on ctDNA samples. In our cohort, detection of $E G F R^{\mathrm{T} 790 \mathrm{M}}$ by NGS at osimertinib relapse had no prognostic value in term of TTD2 ( $\mathrm{P}=0.34$, Table 3). Interestingly, however, patients who developed EGFRdependent mechanisms of resistance responded for longer to third-line treatment $(\mathrm{P}=0.021, \log$-rank test; Figure $3 A, 3 B)$. In addition, a longer OS was observed in patients who developed an EGFR-dependent mechanisms of resistance (median OS 80.5 months) than in patients who did not (40.8 months), even if it did not reach significance ( $\mathrm{P}=0.087$; log-rank test; Figure 3 C).

\section{Discussion}

Liquid biopsy has recently emerged as an essential tool in the management of lung cancer, and more specifically, in treatment monitoring. Indeed, its use in the clinic may reflect tumor heterogeneity, represents a noninvasive and 
Table 3 Univariate analysis of the prognostic value of alterations detected by ctDNA NGS and clinical parameters with regard to TTD2

\begin{tabular}{|c|c|c|c|c|}
\hline & $\begin{array}{l}\text { Number of } \\
\text { samples }\end{array}$ & \multicolumn{3}{|c|}{ Univariate analysis } \\
\hline Age at diagnosis $(<60 ; \geq 60$-year-old) & 17 & 0.43 & $0.12-1.55$ & NS $(0.18)$ \\
\hline Smoking history (Have smoked; Non-smokers) & 17 & 0.40 & $0.10-1.53$ & NS $(0.17)$ \\
\hline$E G F R^{T 790 \mathrm{M}}$ mutation (Positive, Negative) & 17 & 1.90 & $0.49-7.32$ & NS (0.34) \\
\hline Mechanism of resistance (EGFR-dependent; EGFR-independent) & 17 & 4.17 & $1.14-15.30$ & 0.021 \\
\hline
\end{tabular}

Significance (log-rank test) was considered at $\mathrm{P}<0.05$. ctDNA, circulating tumor DNA; NGS, next-generation sequencing; TTD2, time to treatment discontinuation 2; NS, not significant; $\mathrm{HR}$, hazard ratio; $95 \% \mathrm{Cl}, 95 \%$ confidence interval; EGFR, epidermal growth factor receptor.

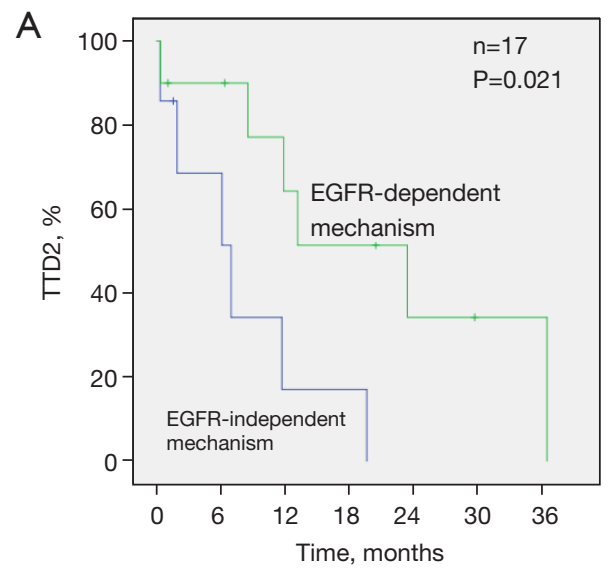

C

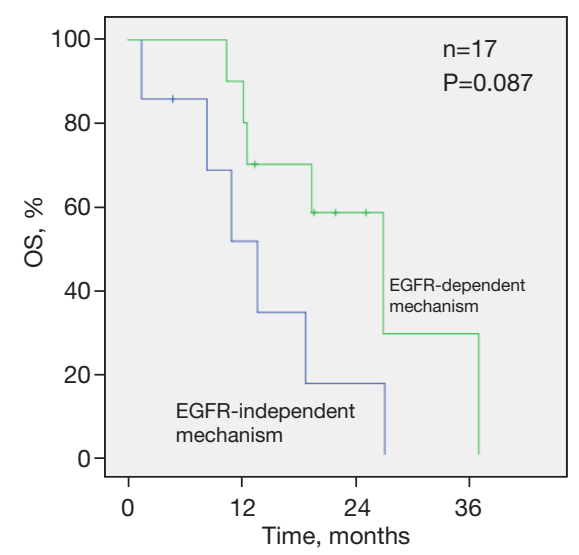

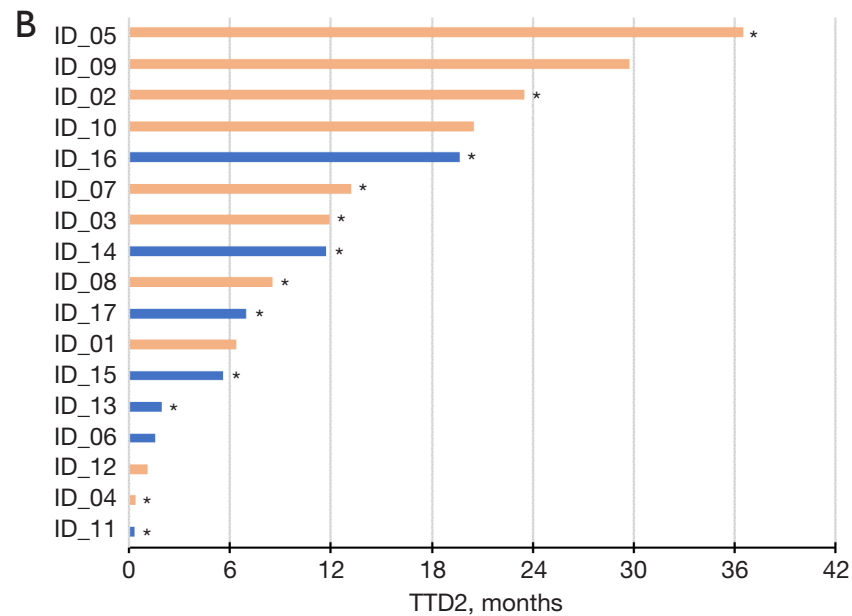

Figure 3 Prognostic value for patients who developed EGFR-dependent and EGFR-independent mechanisms of resistance, as determined by ctDNA NGS analysis. (A) Kaplan-Meier analysis for TTD2. (B) Representation of TTD2 (months) for each patient. The orange bars represented patients who developed an EGFR-dependent mechanism of resistance and the blue bars, patients who developed an EGFRindependent mechanism. *, death. (C) Kaplan-Meier analysis for OS. ctDNA, circulating tumor DNA; NGS, next-generation sequencing; TTD2, time to treatment discontinuation 2; EGFR, epidermal growth factor receptor; OS, overall survival. 
powerful method to evaluate treatment efficiency and offers an opportunity in case of tumor biopsy failure, particularly at relapse (22). Different technical approaches for ctDNA detection and analysis have been specifically adapted to detect mutations at low level. In our study, we used an NGS approach based on the assignment of a molecular barcode to each template DNA molecule, analyzed with an adapted bioinformatic pipeline that allowed error correction. Recently, a multi-laboratory study evaluating different ctDNA NGS assays demonstrated that the approach selected in our study allows detection of mutations with variant allele frequency (VAF) as low as $0.125 \%$, with good correlation of observed to expected VAF (23). Moreover, ctDNA NGS assays have shown good ability to detect multiple actionable mutations, making it a convincing alternative to ddPCR for clinical management of advanced NSCLC (24).

The $E G F R^{\mathrm{T} 790 \mathrm{M}}$ mutation is a robust biomarker, detected in around $50-60 \%$ of patients who developed resistance to first- or second-generation TKIs, that predicts response to osimertinib treatment $(2,25,26)$. Oxnard et al. observed that patients who have lost $E G F R^{\mathrm{T} 790 \mathrm{M}}$ in tissue samples at relapse had a shorter TTD under osimertinib than patients with persisting $E G F R^{\mathrm{T} 790 \mathrm{M}}$ (13). Concordantly, Mehlman et al. showed that the loss of $E G F R^{\mathrm{T} 790 \mathrm{M}}$ at progression was also associated with slightly but not significantly shorter TTD based on the combinatory analysis of tissue samples and a small fraction of ctDNA samples (18). In our study, we observed, to our knowledge for the first time, that the loss of $E G F R^{\mathrm{T} 790 \mathrm{M}}$ based on ctDNA NGS analysis alone was significantly associated with a shorter median TTD, strengthening the clinical utility of NGS from plasma samples. Of note, $E G F R^{\mathrm{C} 797 \mathrm{~S}}$ in conjunction with $E G F R^{\mathrm{T} 790 \mathrm{M}}$ as well as the original $E G F R^{\text {sensitizing }}$ mutation (notated as sensitizing+/T790M+/C797S+ in several reports) has been proposed to better differentiate patients with different outcome $(26,27)$. However, in our cohort, this "triplet pattern" was found in only one case, and therefore could not be evaluated statistically. When we took into account all of the EGFR alterations detected by ctDNA NGS, we could classify patients into those with EGFR-dependent and EGFR-independent mechanisms of resistance to osimertinib. Patients who developed an EGFR-dependent mechanism of resistance responded for longer to osimertinib and also presented a longer post-progression outcome. Of particular interest, in our cohort this signature represents an independent prognostic marker that is more informative than $E G F R^{\mathrm{T} 790 \mathrm{M}}$ status at relapse. Even if this observation has been previously made by other groups using tissue samples (28), our results confirm the convenience of NGS approaches using ctDNA material as a relevant tool to identify patients with better clinical outcomes. Several studies reported that patients who developed an EGFRindependent mechanism of resistance, also called off-target resistance, presented a less durable response to osimertinib that could be assigned to the emergence of a preexisting tumoral subclones under selection pressure of treatment. On the other hand, EGFR-dependent mechanism may result from the acquisition of a novel EGFR mutation that arise after a longer period of treatment and are associated with a more indolent disease $(13,21,28,29)$.

Profiling of plasma with NGS represents a valuable approach for identifying alternative potentially targetable resistance mechanisms, such as $B R A F$ and PIK3CA mutations or MET amplification (30,31). Moreover, its use may bypass problems of tumor heterogeneity by revealing alterations carried only by metastatic sites (32-35). According to our data, PIK3CA $A^{\mathrm{E} 545 \mathrm{~K}}$ mutation was observed in 2 out of 17 patients (11.8\%). Activating PIK3CA mutations are of particular interest as PIK3CA mutant-driven resistance could be overcome by combination treatment with PI3K pathway inhibitors in vitro, providing a possible rationale for a combination treatment (16). We also detected a $K R A S^{\mathrm{G} 12 \mathrm{C}}$ alteration in one case. A phase 1 clinical trial involving patients with NSCLC harboring this mutation has recently reported that patients responded well to sotorasib, offering new opportunities in the treatment of these patients with refractory disease $(36,37) . B R A F^{\mathrm{V} 600 \mathrm{E}}$ was observed in one case. Our team recently reported that one patient harboring this alteration benefited from sequential treatment with dabrafetinib/trametinib and osimertinib (38). Two patients with a MET amplification were also identified by ctDNA NGS and could have benefited from a combination of crizotinib and osimertinib to overcome acquired resistance (39). ERBB2 $2^{\mathrm{G} 776 \mathrm{delinsVC}}$ was also detected, and results from a recent phase II basket trial showed that patients harboring this kind of mutation may benefit from adotrastuzumab emtansine treatment (40). Interestingly, we were able to identify a $E G F R^{\mathrm{C} 797 \mathrm{~S}}$ in cis of the $E G F R^{\mathrm{T} 790 \mathrm{M}}$ mutation that has been shown to respond to combined therapy with brigatinib and cetuximab in a cohort of 15 patients (41). Our ctDNA NGS analyses also reveal presence of other EGFRresistant mutations, including $E G F R^{\mathrm{G} 796 \mathrm{~S}}$ cis-oriented with $E G F R^{\mathrm{T} 790 \mathrm{M}}$, and $E G F R^{L 718 Q}, E G F R^{E 758 D}$, and $E G F R^{A 859 S}$ cisoriented with $E G F R^{L 858 R}$. Even if these alterations have been previously reported in osimertinib-resistant patients, no 
targeted therapy is currently available for these $(28,42,43)$. Finally, although ctDNA exploration is not the most appropriate material to highlight histological transformation that occurs in around $15 \%$ of patients receiving osimertinib as a later-line therapy (21), our results and those of others show that early detection of alterations in RB1 and TP53 genes in plasma samples might reflect the acquisition of this mechanism of resistance $(38,44)$.

Regarding our study, it is important to mention that the small sample size of our cohort is a significant limitation and therefore, even if our results are of particular interest, they should be further validated in an independent cohort of appropriate size. In addition, corresponding tissue samples or ctDNA samples obtained before initiation of osimertinib treatment were only available for 5 and 8 patients, respectively. Unfortunately, this prevented complementary NGS and statistical analyses that could have been performed to determine whether the mechanism of resistance identified after relapse under osimertinib emerged from the selection of a preexisting resistant clone or from the de novo acquisition of novel alteration. This information would have been of particular interest for patients who developed a histological transformation as it has been shown that NSCLC samples, that carried an $E G F R^{\text {activating }}$ concomitantly with TP53 and RB1 alterations before initiation of TKI treatment, have a higher risk to develop this resistant mechanism $(44,45)$.

Although NGS approaches are not the most sensitive techniques to detect mutations in plasma samples (22), our results reveal their powerful utility in routine clinical care of lung cancer patients, notably to highlight a broad panel of alterations for detection of novel acquired resistance and tumor heterogeneity. Despite the limitations reported, we hope that our work demonstrates the importance of ctDNA NGS for detection of resistance mechanisms during treatment, making it a potentially valuable tool to rapidly adopt the appropriate drug after osimertinib failure.

\section{Acknowledgments}

The authors thank the patients, donors, and their kin for agreement to publication of the report.

Funding: None.

\section{Footnote}

Reporting Checklist: The authors have completed the MDAR reporting checklist. Available at https://dx.doi.org/10.21037/ tlcr-21-679

Data Sharing Statement: Available at https://dx.doi. org/10.21037/tlcr-21-679

Conflicts of Interest: All authors have completed the ICMJE uniform disclosure form (available at https://dx.doi. org/10.21037/tlcr-21-679). The authors have no conflicts of interest to declare.

Ethical Statement: The authors are accountable for all aspects of the work in ensuring that questions related to the accuracy or integrity of any part of the work are appropriately investigated and resolved. The study was conducted in accordance with the Declaration of Helsinki (as revised in 2013). The study was approved by the Institutional Review Board of the University Hospital of Montpellier (IRBMTP_2020_09_202000581). An approved informed consent statement was acquired for all patients.

Open Access Statement: This is an Open Access article distributed in accordance with the Creative Commons Attribution-NonCommercial-NoDerivs 4.0 International License (CC BY-NC-ND 4.0), which permits the noncommercial replication and distribution of the article with the strict proviso that no changes or edits are made and the original work is properly cited (including links to both the formal publication through the relevant DOI and the license). See: https://creativecommons.org/licenses/by-nc-nd/4.0/.

\section{References}

1. Soria JC, Ohe Y, Vansteenkiste J, et al. Osimertinib in Untreated EGFR-Mutated Advanced Non-Small-Cell Lung Cancer. N Engl J Med 2018;378:113-25.

2. Mok TS, Wu YL, Ahn MJ, et al. Osimertinib or PlatinumPemetrexed in EGFR T790M-Positive Lung Cancer. N Engl J Med 2017;376:629-40.

3. Goss G, Tsai CM, Shepherd FA, et al. Osimertinib for pretreated EGFR Thr790Met-positive advanced nonsmall-cell lung cancer (AURA2): a multicentre, open-label, single-arm, phase 2 study. Lancet Oncol 2016;17:1643-52.

4. Ramalingam SS, Yang JC, Lee CK, et al. Osimertinib As First-Line Treatment of EGFR Mutation-Positive Advanced Non-Small-Cell Lung Cancer. J Clin Oncol 2018;36:841-9.

5. Yang JC, Ahn MJ, Kim DW, et al. Osimertinib in 
Pretreated T790M-Positive Advanced Non-SmallCell Lung Cancer: AURA Study Phase II Extension Component. J Clin Oncol 2017;35:1288-96.

6. Ortiz-Cuaran S, Scheffler M, Plenker D, et al. Heterogeneous Mechanisms of Primary and Acquired Resistance to Third-Generation EGFR Inhibitors. Clin Cancer Res 2016;22:4837-47.

7. Ou SI, Agarwal N, Ali SM. High MET amplification level as a resistance mechanism to osimertinib (AZD9291) in a patient that symptomatically responded to crizotinib treatment post-osimertinib progression. Lung Cancer 2016;98:59-61.

8. Planchard D, Loriot Y, André F, et al. EGFR-independent mechanisms of acquired resistance to AZD9291 in EGFR T790M-positive NSCLC patients. Ann Oncol 2015;26:2073-8.

9. Yu HA, Tian SK, Drilon AE, et al. Acquired Resistance of EGFR-Mutant Lung Cancer to a T790M-Specific EGFR Inhibitor: Emergence of a Third Mutation (C797S) in the EGFR Tyrosine Kinase Domain. JAMA Oncol 2015;1:982-4.

10. Beagan JJ, Bach S, van Boerdonk RA, et al. Circulating tumor DNA analysis of EGFR-mutant non-small cell lung cancer patients receiving osimertinib following previous tyrosine kinase inhibitor treatment. Lung Cancer 2020;145:173-80.

11. Buder A, Hochmair MJ, Setinek U, et al. EGFR mutation tracking predicts survival in advanced EGFR-mutated nonsmall cell lung cancer patients treated with osimertinib. Transl Lung Cancer Res 2020;9:239-45.

12. Del Re M, Bordi P, Rofi E, et al. The amount of activating EGFR mutations in circulating cell-free DNA is a marker to monitor osimertinib response. Br J Cancer 2018;119:1252-8.

13. Oxnard GR, Hu Y, Mileham KF, et al. Assessment of Resistance Mechanisms and Clinical Implications in Patients With EGFR T790M-Positive Lung Cancer and Acquired Resistance to Osimertinib. JAMA Oncol 2018;4:1527-34.

14. Provencio M, Torrente M, Calvo V, et al. Prognostic value of quantitative ctDNA levels in non small cell lung cancer patients. Oncotarget 2018;9:488-94.

15. Usui K, Yokoyama T, Naka G, et al. Plasma ctDNA monitoring during epidermal growth factor receptor (EGFR)-tyrosine kinase inhibitor treatment in patients with EGFR-mutant non-small cell lung cancer (JP-CLEAR trial). Jpn J Clin Oncol 2019;49:554-8.

16. Vaclova T, Grazini U, Ward L, et al. Clinical impact of subclonal EGFR T790M mutations in advanced-stage EGFR-mutant non-small-cell lung cancers. Nat Commun 2021;12:1780.

17. Eisenhauer EA, Therasse P, Bogaerts J, et al. New response evaluation criteria in solid tumours: revised RECIST guideline (version 1.1). Eur J Cancer 2009;45:228-47.

18. Mehlman C, Cadranel J, Rousseau-Bussac G, et al. Resistance mechanisms to osimertinib in EGFR-mutated advanced nonsmall-cell lung cancer: A multicentric retrospective French study. Lung Cancer 2019;137:149-56.

19. Le X, Puri S, Negrao MV, et al. Landscape of EGFRDependent and -Independent Resistance Mechanisms to Osimertinib and Continuation Therapy Beyond Progression in EGFR-Mutant NSCLC. Clin Cancer Res 2018;24:6195-203.

20. Schmid S, Li JJN, Leighl NB. Mechanisms of osimertinib resistance and emerging treatment options. Lung Cancer 2020;147:123-9.

21. Schoenfeld AJ, Chan JM, Kubota D, et al. Tumor Analyses Reveal Squamous Transformation and Off-Target Alterations As Early Resistance Mechanisms to First-line Osimertinib in EGFR-Mutant Lung Cancer. Clin Cancer Res 2020;26:2654-63.

22. Vendrell JA, Mau-Them FT, Béganton B, et al. Circulating Cell Free Tumor DNA Detection as a Routine Tool forLung Cancer Patient Management. Int J Mol Sci 2017;18:264.

23. He HJ, Stein EV, Konigshofer Y, et al. Multilaboratory Assessment of a New Reference Material for Quality Assurance of Cell-Free Tumor DNA Measurements. J Mol Diagn 2019;21:658-76.

24. Rolfo C, Mack PC, Scagliotti GV, et al. Liquid Biopsy for Advanced Non-Small Cell Lung Cancer (NSCLC): A Statement Paper from the IASLC. J Thorac Oncol 2018;13:1248-68.

25. Jenkins S, Yang JC, Ramalingam SS, et al. Plasma ctDNA Analysis for Detection of the EGFR T790M Mutation in Patients with Advanced Non-Small Cell Lung Cancer. J Thorac Oncol 2017;12:1061-70.

26. Lin CC, Shih JY, Yu CJ, et al. Outcomes in patients with non-small-cell lung cancer and acquired Thr790Met mutation treated with osimertinib: a genomic study. Lancet Respir Med 2018;6:107-16.

27. Romero A, Serna-Blasco R, Alfaro C, et al. ctDNA analysis reveals different molecular patterns upon disease progression in patients treated with osimertinib. Transl Lung Cancer Res 2020;9:532-40.

28. Mu Y, Hao X, Xing P, et al. Acquired resistance to 
osimertinib in patients with non-small-cell lung cancer: mechanisms and clinical outcomes. J Cancer Res Clin Oncol 2020;146:2427-33.

29. Oxnard GR, Arcila ME, Sima CS, et al. Acquired resistance to EGFR tyrosine kinase inhibitors in EGFRmutant lung cancer: distinct natural history of patients with tumors harboring the T790M mutation. Clin Cancer Res 2011;17:1616-22.

30. Leonetti A, Sharma S, Minari R, et al. Resistance mechanisms to osimertinib in EGFR-mutated non-small cell lung cancer. Br J Cancer 2019;121:725-37.

31. Papadimitrakopoulou VA, Wu YL, Han JY, et al. Analysis of resistance mechanisms to osimertinib in patients with EGFR T790M advanced NSCLC from the AURA3 study. Ann Oncol 2018;29:741.

32. Solassol J, Vendrell JA, Senal R, et al. Challenging BRAF/ EGFR co-inhibition in NSCLC using sequential liquid biopsies. Lung Cancer 2019;133:45-7.

33. Dono A, Takayasu T, Yan Y, et al. Differences in Genomic Alterations Between Brain Metastases and Primary Tumors. Neurosurgery 2021;88:592-602.

34. Liu C, Liu C, Zou X, et al. Next-generation sequencing facilitates differentiating between multiple primary lung cancer and intrapulmonary metastasis: a case series. Diagn Pathol 2021;16:21.

35. Gremel G, Lee RJ, Girotti MR, et al. Distinct subclonal tumour responses to therapy revealed by circulating cellfree DNA. Ann Oncol 2016;27:1959-65.

36. Canon J, Rex K, Saiki AY, et al. The clinical KRAS(G12C) inhibitor AMG 510 drives anti-tumour immunity. Nature 2019;575:217-23.

37. Hong DS, Fakih MG, Strickler JH, et al. KRASG12C Inhibition with Sotorasib in Advanced Solid Tumors. N Engl J Med 2020;383:1207-17.

Cite this article as: Vendrell JA, Quantin X, Aussel A, Solassol I, Serre I, Solassol J. EGFR-dependent mechanisms of resistance to osimertinib determined by ctDNA NGS analysis identify patients with better outcome. Transl Lung Cancer Res 2021;10(11):4084-4094. doi: 10.21037/tlcr-21-679
38. Vendrell JA, Quantin X, Serre I, et al. Combination of tissue and liquid biopsy molecular profiling to detect transformation to small cell lung carcinoma during osimertinib treatment. Ther Adv Med Oncol 2020;12:1758835920974192.

39. York ER, Varella-Garcia M, Bang TJ, et al. Tolerable and Effective Combination of Full-Dose Crizotinib and Osimertinib Targeting MET Amplification Sequentially Emerging after T790M Positivity in EGFR-Mutant NonSmall Cell Lung Cancer. J Thorac Oncol 2017;12:e85-8.

40. Li BT, Shen R, Buonocore D, et al. Ado-Trastuzumab Emtansine for Patients With HER2-Mutant Lung Cancers: Results From a Phase II Basket Trial. J Clin Oncol 2018;36:2532-7.

41. Wang Y, Yang N, Zhang Y, et al. Effective Treatment of Lung Adenocarcinoma Harboring EGFR-Activating Mutation, T790M, and cis-C797S Triple Mutations by Brigatinib and Cetuximab Combination Therapy. J Thorac Oncol 2020;15:1369-75.

42. Lin L, Lu Q, Cao R, et al. Acquired rare recurrent EGFR mutations as mechanisms of resistance to Osimertinib in lung cancer and in silico structural modelling. Am J Cancer Res 2020;10:4005-15.

43. Yang $Y$, Zhang X, Wang R, et al. Osimertinib Resistance With a Novel EGFR L858R/A859S/Y891D Triple Mutation in a Patient With Non-Small Cell Lung Cancer: A Case Report. Front Oncol 2020;10:542277.

44. Offin M, Chan JM, Tenet M, et al. Concurrent RB1 and TP53 Alterations Define a Subset of EGFR-Mutant Lung Cancers at risk for Histologic Transformation and Inferior Clinical Outcomes. J Thorac Oncol 2019;14:1784-93.

45. Popat S. Histologically Transformed SCLC From EGFRMutant NSCLC: Understanding the Wolf in Sheep's Clothing. J Thorac Oncol 2019;14:1689-91. 
Supplementary

Table S1 The LiquidPlex ${ }^{\mathrm{TM}}$ 28-gene panel (ArcherDx)

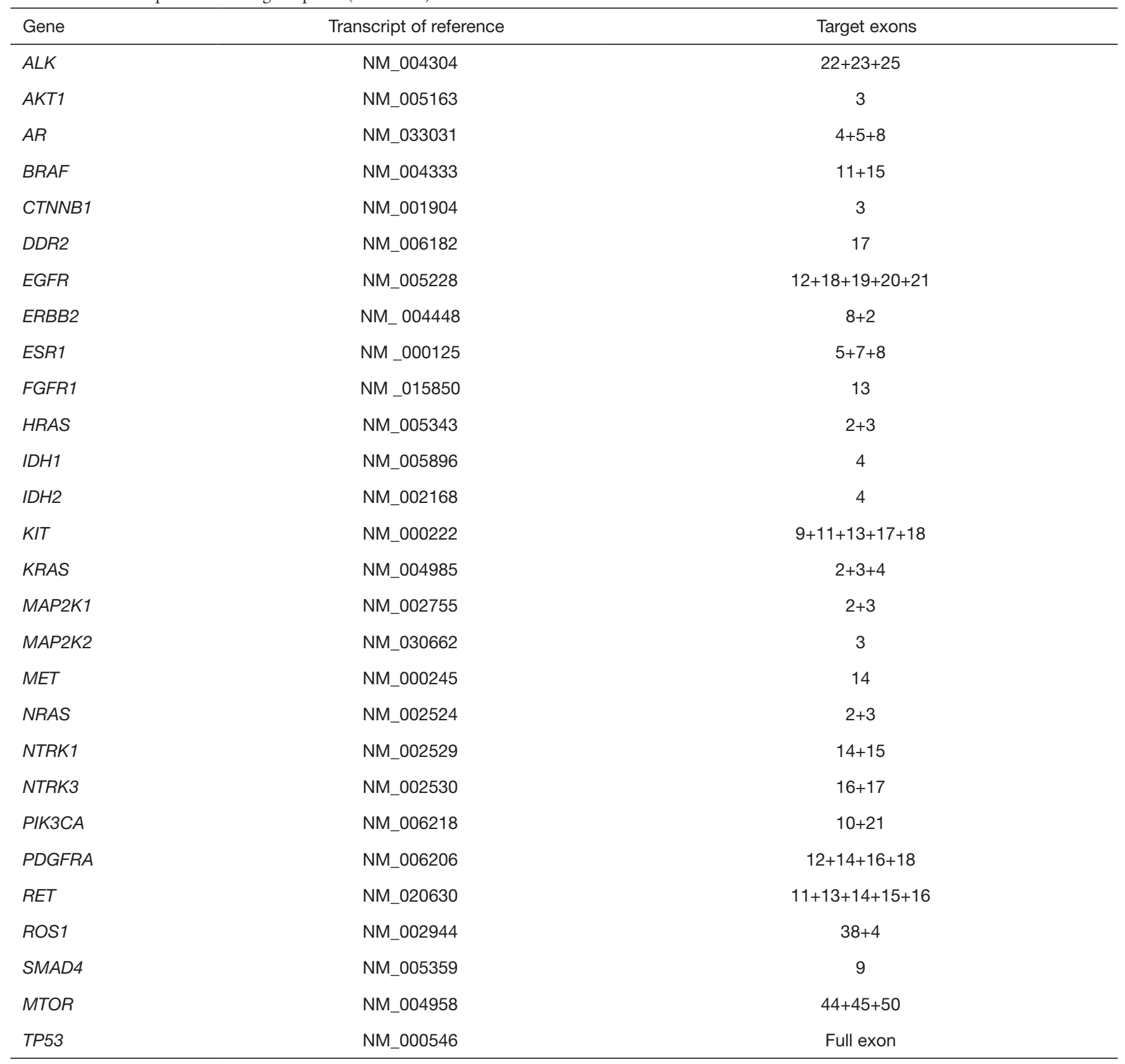


A

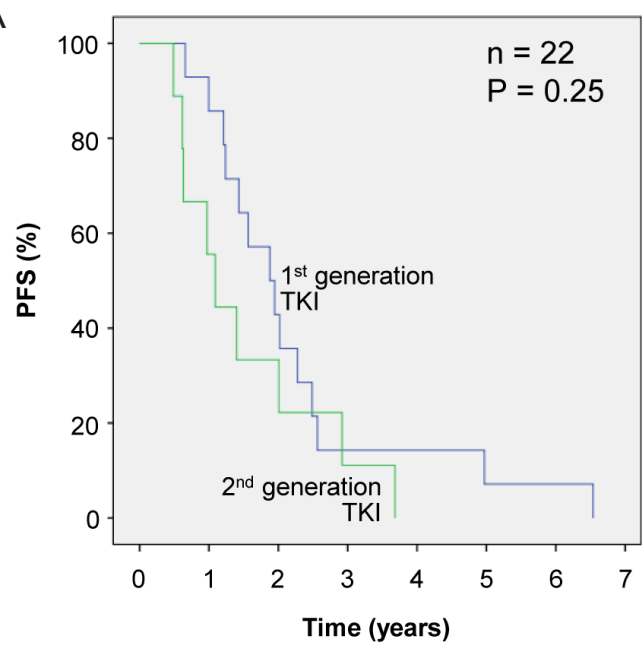

B

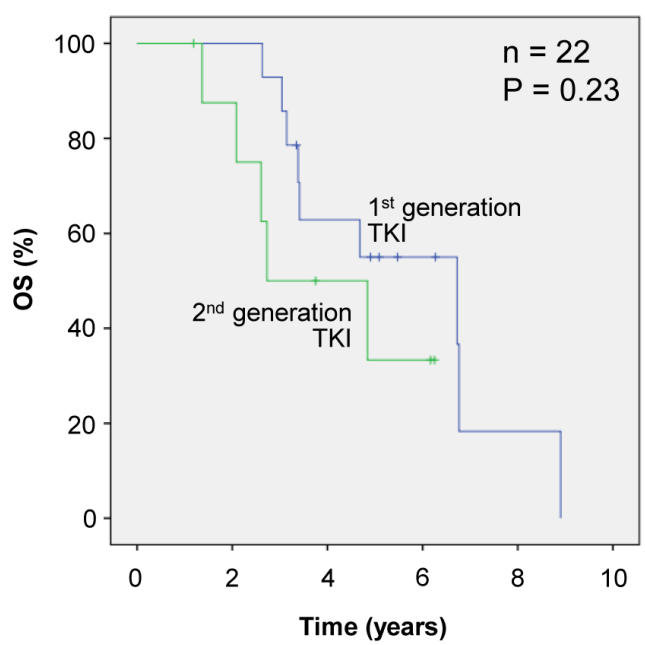

Figure S1 Kaplan-Meier analyses of patients who received first- or second-generation TKI as first-line treatment in terms of PFS (A) and OS (B). TKI, tyrosine kinase inhibitor; PFS, progression-free survival; OS, overall survival. 
Table S2 Statistical comparison of the clinical characteristics in the patients who developed an EGFR-dependent or -independent mechanism of resistance $(\mathrm{n}=17)$

\begin{tabular}{|c|c|c|c|}
\hline \multicolumn{4}{|l|}{ Sex } \\
\hline Male & 3 & 5 & NS (0.15) \\
\hline Female & 7 & 2 & \\
\hline \multicolumn{4}{|l|}{ Age } \\
\hline$<60$ & 5 & 3 & NS (1) \\
\hline$\geq 60$ & 5 & 4 & \\
\hline \multicolumn{4}{|l|}{ Smoking status } \\
\hline Have smoked & 4 & 2 & NS (1) \\
\hline$E G F R^{\text {del19 }}$ & 5 & 4 & NS $(0.41)$ \\
\hline$E G F R^{\mathrm{L} 858 \mathrm{R}}$ & 4 & 1 & \\
\hline$E G F R^{\mathrm{L} 861 \mathrm{Q}}$ & 1 & 2 & \\
\hline \multicolumn{4}{|l|}{$1^{\text {st }}$ line TKI treatment } \\
\hline $1^{\text {st }}$ generation TKI & 8 & 4 & NS (0.59) \\
\hline $2^{\text {nd }}$ generation TKI & 2 & 3 & \\
\hline \multicolumn{4}{|c|}{ Presence of metastases at osimertinib treatment initiation } \\
\hline
\end{tabular}

Significance (Fisher exact test) was considered at $\mathrm{P}<0.05$. NS, not significant. 